\title{
Guidelines for the Li-Fraumeni and heritable TP53-related cancer syndromes
}

\author{
Thierry Frebourg ${ }^{1} \cdot$ Svetlana Bajalica Lagercrantz ${ }^{2} \cdot$ Carla Oliveira $^{3} \cdot$ Rita Magenheim $^{4} \cdot$ D. Gareth Evans $\mathbb{D}^{5} \cdot$ \\ The European Reference Network GENTURIS
}

Received: 11 February 2020 / Revised: 28 March 2020 / Accepted: 8 April 2020 / Published online: 26 May 2020

(c) The Author(s) 2020. This article is published with open access

\begin{abstract}
Fifty years after the recognition of the Li-Fraumeni syndrome (LFS), our perception of cancers related to germline alterations of TP53 has drastically changed: (i) germline TP53 alterations are often identified among children with cancers, in particular soft-tissue sarcomas, adrenocortical carcinomas, central nervous system tumours, or among adult females with early breast cancers, without familial history. This justifies the expansion of the LFS concept to a wider cancer predisposition syndrome designated heritable TP53-related cancer (hTP53rc) syndrome; (ii) the interpretation of germline TP53 variants remains challenging and should integrate epidemiological, phenotypical, bioinformatics prediction, and functional data; (iii) the penetrance of germline disease-causing TP53 variants is variable, depending both on the type of variant (dominantnegative variants being associated with a higher cancer risk) and on modifying factors; (iv) whole-body MRI (WBMRI) allows early detection of tumours in variant carriers and (v) in cancer patients with germline disease-causing TP53 variants, radiotherapy, and conventional genotoxic chemotherapy contribute to the development of subsequent primary tumours. It is critical to perform TP53 testing before the initiation of treatment in order to avoid in carriers, if possible, radiotherapy and genotoxic chemotherapies. In children, the recommendations are to perform clinical examination and abdominal ultrasound every 6 months, annual WBMRI and brain MRI from the first year of life, if the TP53 variant is known to be associated with childhood cancers. In adults, the surveillance should include every year clinical examination, WBMRI, breast MRI in females from 20 until 65 years and brain MRI until 50 years.
\end{abstract}

Members of the European Reference Network GENTURIS are listed below Acknowledgements.

Thierry Frebourg

frebourg@chu-rouen.fr

1 Department of Genetics, Rouen University Hospital and Inserm U1245, Normandie University, UNIROUEN, Normandy Centre for Genomic and Personalized Medicine, Rouen, France

2 Hereditary Cancer Unit, Department of Clinical Genetics, Karolinska University Hospital, Stockholm, Sweden

3 i3S-Instituto de Investigação e Inovação em Saúde \& Institute of Molecular Pathology and Immunology of the University of Porto, and Porto Comprehensive Cancer Center, Porto, Portugal

4 Community Representative, Berlin, Germany

5 Manchester Centre for Genomic Medicine, Division of Evolution and Genomic Sciences, University of Manchester, MAHSC, St Mary's Hospital, Manchester University Hospitals NHS Foundation Trust, Manchester, UK

\section{Introduction}

\section{From Li-Fraumeni syndrome to heritable TP53-related cancers}

Heterozygous germline TP53 alterations were identified in 1990 in the Li-Fraumeni syndrome (LFS), described in 1969 by Frederick Li and Joseph Fraumeni [1-3]. The p53 protein normally acts as a guardian of the genome, and if DNA damage occurs, p53 triggers a response based on transcription regulation of numerous genes involved in cell cycle, DNA repair, apoptosis, senescence and cellular metabolism. LFS is characterised by a strong familial aggregation of cancers, early-onset of tumours and wide tumour spectrum, including the so-called core LFS cancers: soft-tissue sarcomas (STS), osteosarcomas, adrenocortical carcinomas (ACC), central nervous system (CNS) tumours and very early-onset female breast cancers, occurring before 31 years. Fifty years after the initial clinical recognition of the syndrome, germline alterations of TP53 are mainly 
identified among children with cancers or among adult females with breast cancers, in both cases often without familial history of cancer. For this reason, our perception of cancers related to germline alterations of TP53 has drastically changed through time [4-6]. The diversity of clinical presentations associated with germline TP53 alterations justifies the expansion of the LFS concept to a wider cancer predisposition syndrome designated heritable TP53-related cancer (hTP53rc) syndrome. Criteria for germline TP53 variant screening named 'Chompret criteria' have been sequentially updated ([6]; see below recommendation 1). Regardless of familial history, the detection rate of disease causing germline TP53 variants has been estimated to be: 50-80\% in children presenting with ACC [6-9] or choroid plexus carcinomas $[6,10]$; up to $73 \%$ in children with rhabdomyosarcoma of embryonal anaplastic subtype [11] and between 3.8 and $7.7 \%$ in females with breast carcinoma before 31 years of age [12]. These data demonstrate that familial history of cancer should not be mandatory when considering genetic testing of TP53. The frequency of presentations without familial cancer history is explained both by the contribution of de novo variants to hTP53rc syndrome, which has been estimated to be between 7 and $20 \%[13,14]$, and the incomplete penetrance of germline TP53 variants. Beside the Chompret criteria, recent reports and experience of certain centres justify to extend TP53 testing to other clinical presentations suggestive of a germline TP53 alteration: Children and adolescents with hypodiploid acute lymphoblastic leukaemia [15, 16], otherwise unexplained sonic hedgehog-driven medulloblastoma [17], jaw osteosarcoma (see below recommendation 2) and patients who develop a second primary tumour within the radiotherapy field of a first core TP53 tumour which occurred before 46 years (see below recommendation 3).

\section{Interpretation of germline TP53 variants}

Because the TP53 gene is currently included in several cancer gene panels broadly used in genetic testing, the number of TP53 tests performed in non-suggestive clinical situations has exponentially increased. Whereas the interpretation of TP53 variants predicted to result in loss of function, such as nonsense or frameshift deletions or insertions, is usually obvious, the interpretation of missense variants, representing the majority, is often challenging and requires specific expertise. The most common consequence of germline variants causing hTP53rc syndrome is the functional inactivation of the protein. Classification of TP53 missense variants, in agreement with the ACMG/AMP guidelines, is based on several considerations including phenotypical data (identified in patients fulfilling the Chompret criteria); frequency of the variant in the general population, as reported by the Genome Aggregation Database (https://gnomad.broadinstitute.org/), bioinformatics predictions of the variant impact on protein or RNA splicing using different algorithms, and functional analyses of the variants performed using different in vitro assays performed either in yeast or cultured cells [18-21] (http://p53. iarc.fr/). Optimised and stringent ACMG/AMP criteria for a specific classification of germline TP53 variants, integrating the above considerations, are being developed by a TP53 variant curation expert panel, under the umbrella of ClinGen. This will allow a progressive allocation or reclassification of TP53 variants into the different ACMG/ AMP classes. Since the distinction between class 5 (pathogenic) and class 4 (likely pathogenic) variants is particularly subtle for TP53 variants, these variants are designated in the current ERN guideline as 'disease-causing' variants.

\section{The question of mosaicism}

The presence of mosaic TP53 alterations should be considered in patients with sporadic cancers strongly suggestive of a disease-causing TP53 variant, such as childhood ACC, choroid plexus carcinoma, and breast cancer before 31 years of age and in patients with multiple primary tumours belonging to the TP53 core tumour spectrum [14]. The absence of detectable TP53 variants after analysis of blood DNA using NGS, even performed at a high depth, does not guarantee the absence of mosaic alterations which can be restricted to other tissues than blood. Therefore, a complete screening for TP53 disease causing variants in highly suggestive situations should include analysis of tumour tissue, which is so far not systematically performed. In contrast, the detection of a TP53 variant in a small fraction of NGS reads from blood DNA does not always correspond to a mosaic alteration [22-25] and molecular geneticists should be aware of two pitfalls: the first corresponds to circulating tumour DNA, commonly observed in patients with metastatic cancers. For instance, the detection of a TP53 variant in the blood from a patient with metastatic high grade serous ovary carcinoma is likely to correspond to circulating tumour DNA, considering the very high frequency of somatic TP53 alterations in these malignancies ( $>95 \%)$; the second is due to clonal haematopoiesis, corresponding to the occurrence, in hematopoietic stem cells of somatic TP53 alterations conferring a growth advantage. Clonal haematopoiesis was initially reported in patients over 70 years of age, but can be detected from 30 years of age. The frequency of clonal haematopoiesis is increasing with age, tobacco use and exposure to chemotherapy or radiotherapy [22-25]. Therefore, when a TP53 variant is detected in a small fraction of NGS reads from blood, it is critical, before concluding that the presence represents a mosaic TP53 
alteration, to respect the following rules: (i) consider the clinical presentation (suggestive or not of the presence of a disease-causing TP53 variant) and medical history (treatments, metastases, etc...) and (ii) confirm the presence of the variant in the tissue from which the tumour originated. Further confirmation in an unaffected tissue with no lymphocyte content, such as a hair follicle, skin biopsy or nail clippings, should also be considered if circulating tumour DNA is suspected from metastatic disease.

\section{Cancer risk associated with germline TP53 variants}

A challenge when dealing with TP53 variant carriers is to estimate the cancer risk or penetrance associated with each specific TP53 variant, and this cancer risk has recently been revisited. Indeed, the cumulative cancer incidence of germline disease-causing TP53 variants was initially calculated using information mainly from familial cases and was estimated to $73-100 \%$ by age 70 , with risks close to $100 \%$ in women [26-28]. The predominance of familial cases likely results in an ascertainment bias and an overestimation of disease penetrance. This should be regarded in perspective with the prevalence in the general population of germline disease-causing TP53 variants, which was recently estimated, based on a conservative approach, to be in the magnitude of 1 among 4500 individuals [29]. In childhood, the main tumour risks are ACC, STS, osteosarcomas and CNS tumours whereas the main tumour risk in adults corresponds to female breast cancers, female TP53 variant carriers have an excessively high risk of developing breast cancer before 31 . There is no known elevated risk of male breast cancer [4-6, 26-28, 30]. There is a perception that colorectal cancer is associated with germline pathogenic TP53 variants [31-33]. However, the corresponding studies suffer from methodological limitations and interpretation of some reported TP53 variants is problematic. Families with a germline TP53 variant and an additional history of colorectal cancer in the pedigree may have increased risk of colorectal cancer. This increased risk is, however, not associated with the TP53 variant itself and, on the basis of the published studies, a high risk of colorectal cancer can be confidently excluded in carriers of disease-causing TP53 variants.

The penetrance of germline disease-causing TP53 variants is variable. One factor explaining the variability of this penetrance is the type of the variant itself: some of the p53 proteins bearing missense mutations are classified as dominant-negative due to their ability to complex and reduce the transcriptional activity of wild-type $\mathrm{p} 53$ protein, producing malfunctioning or non-functioning p53 tetramers. These dominant-negative missense TP53 variants are usually detected in families with childhood cancers and are generally highly penetrant. In contrast, null variants (frameshift or nonsense variants, splicing variants, large genomic rearrangements, and non-dominant-negative missense variants), are predominantly identified in families with mostly adult cancers and have a lower disease penetrance [6]. A remarkable example of a low penetrant, but still disease-causing variant, is the non-dominant-negative missense p.Arg337His variant, present in $0.3 \%$ of the population from Southern Brazil and associated with a founder effect [34-36]. The difference in the clinical severity between dominant-negative missense variants and the remaining ones is explained by a difference in their biological impact on the p 53 transcriptional activity. Indeed, measurement of the transcriptional response to DNA damage in cells harbouring heterozygous TP53 variants, has shown that dominant-negative missense variants have a more drastic impact on p53 DNA binding and transcriptional response to DNA damage, than the other types of heterozygous alterations [19]. The clinical annotation of the variants and updated functional data should allow progressively, dichotomising disease-causing TP53 variants in 'high cancer risk' and 'low cancer risk' alleles.

The phenotypic variability observed within the same family (e.g. a child affected with cancer and the parent, carrier of the same variant, being not affected in childhood) strongly supports the existence of genetic modifying factors and their identification represents, at the present time, a top priority in the field. It is more and more evident that phenotypic expression in carriers of TP53 disease-causing variants is also dependent on environmental factors, as germline TP53 variants may turn p53 into a protein permissive to oncogenic stress.

\section{The impact of radio and chemotherapy on the development of subsequent primary tumours}

Germline TP53 variant carriers have a remarkably high incidence of subsequent primary tumours, which may occur in more than $40 \%$ of TP53 variant carriers [6, 27]. Subsequent primary tumours often develop after the exposure of TP53 variant carriers to radio and/or chemotherapy treatments. The demonstration of the contribution of radiotherapy and conventional chemotherapy to the development of subsequent primary tumours in these carriers came from consistent observations of sequential development of multiple tumours after treatment by chemo- or radiotherapy of a first tumour and the development of tumours within the radiotherapy field [6]. A cause-effect was strongly supported by studies of the impact of chemo- and radiotherapy in lymphocytes with wild-type or mutant TP53 genotype and LFS mouse models [37]. Therefore, in cancer patients, testing for disease-causing TP53 variants must absolutely take place before starting treatment and if a disease-causing TP53 variant is found, priority should be given to surgical 
or ablative treatments, avoiding radiotherapy when possible and using preferably non-genotoxic chemotherapies.

\section{Surveillance protocols}

Surveillance protocols for carriers bearing disease-causing TP53 variants have recently been elaborated in the framework of an international consortium coordinated by Canadian and US teams [38, 39]. These protocols indicate that carriers should undergo abdominal ultrasound every 3-4 months, annual whole-body MRI (WBMRI) and annual brain MRI (the first with gadolinium enhancement) from the first year of life. Additionally, female carriers should undergo annual breast MRI from the age of 20 years onwards. The option of risk-reducing mastectomy may be discussed on a case-by-case basis [39]. Several international studies, mostly performed without gadolinium-based contrast agents (GBCAs), have confirmed the efficiency of WBMRI, with an overall estimated detection rate of $7 \%$ for new and localised primary cancers on a first prevalent screen [40-46]. Given that GBCAs may be retained for months or years in several organs, multiple GBCAs administrations should probably be avoided in germline TP53 variant carriers and only macrocyclic GBCAs, which are apparently less retained in the body [47], should be used.

\section{Scope of the guidelines}

These guidelines have been elaborated by members of the European Reference Network (ERN) on the Genetic Tumour Risk Syndromes (GENTURIS) in order to integrate the available information with clinical utility and to assist health care professionals in the identification and management of patients with germline disease-causing TP53 variants, causing heritable TP53-related cancer (hTP53rc) syndrome. In particular, these guidelines take into account the diversity of clinical presentations associated with germline TP53 variants, the variability of the TP53 variant penetrance, the role of radiotherapy and chemotherapy in the development of subsequent primary tumour and the medical benefit of surveillance protocols. These guidelines do not signify nor intend to be a legal standard of care, they should support clinical decision making, but never replace clinical professionals. This is particularly critical for hTP53rc syndrome, considering the diversity of clinical expression related to germline TP53 variants.

\section{Methods followed}

The ERN GENTURIS hTP53rc syndrome guideline was established by molecular and clinical geneticists and clinicians with expertise in paediatrics, oncology, or radiology, as well as affected individuals and parent representatives.
The Guideline Development Group was supported by a Core Writing Group composed of ERN GENTURIS HCP members from different member states and who are recognised experts and specialised in molecular oncobiology and/or clinical practice and/or in the diagnosis and management of hTP53rc. The Core Writing Group leads had joint meetings with a Patient Advisory Group composed of affected individuals and parent representatives that have experience with hTP53rc syndrome. The elaboration of these guidelines has then actively involved external experts from different speciality areas that are relevant to the scope of the guideline.

The guidelines were elaborated on the basis of 337 published articles extracted from Pubmed, using the following terms: (screening [title/abstract] OR surveillance [title/abstract] OR detection [title/abstract]) AND (LFS[title] OR Li-Fraumeni [Title] OR TP53 [title]) AND 'humans' [MeSH Terms]. Additional articles were requested from experts in the field and references of all the articles were considered. As is typical for many rare diseases, the volume of peer-reviewed evidence available to consider for these guidelines was small and came from a limited number of articles, which typically reported on small samples or series. To balance the weight of both published evidence and quantify the wealth of expert experience and knowledge, we have used for evidence grading the following scale: (i) strong evidence: consistent evidence and new evidence unlikely to change recommendation and expert consensus; (ii) moderate evidence: expert consensus or majority decision but with inconsistent evidence or significant new evidence expected and (iii) weak evidence: inconsistent evidence AND expert agreement.

\section{Recommendations}

\section{Cancer patients who should be tested for germline disease-causing TP53 ${ }^{\mathrm{a}}$}

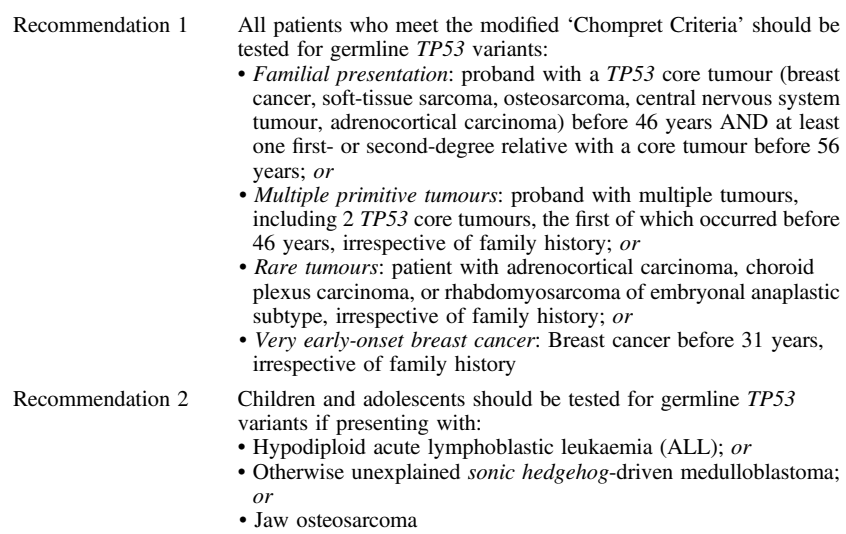
tested for germline TP53 variants:

- Familial presentation: proband with a TP53 core tumour (breast - Familial presentation: proband with a TP53 core tumour (breast
cancer, soft-tissue sarcoma, osteosarcoma, central nervous system tumour, adrenocortical carcinoma) before 46 years AND at least one first- or second-degree relative with a core tumour before 56 years; or

- Multiple primitive tumours: proband with multiple tumours, including 2 TP53 core tumours, the first of which occurred before 46 years, irrespective of family history; or

- Rare tumours: patient with adrenocortical carcinoma, choroid - Rare tumours: patient with adrenocortical carcinoma, choroid
plexus carcinoma, or rhabdomyosarcoma of embryonal anaplastic subtype, irrespective of family history; or

- Very early-onset breast cancer: Breast cancer before 31 years, irrespective of family history

Recommendation 2 Children and adolescents should be tested for germline TP53 variants if presenting with:

- Hypodiploid acute lymphoblastic leukaemia (ALL); or

- Otherwise unexplained sonic hedgehog-driven medulloblastoma; or

- Jaw osteosarcoma 
Table (continued)

Recommendation 3 Patients who develop a second primary tumour, within the radiotherapy field of a first core TP53 tumour which occurre before 46 years, should be tested for germline TP53 variants

Recommendation $4 \quad$ a. Patients older than 46 years presenting with breast cancer without personal or familial history fulfilling the 'Chompret Criteria' should not be tested for germline TP53 variants

b. Any patient presenting with isolated breast cancer and not fulfilling the 'Chompret Criteria', in whom a disease-causing TP53 variant has been identified, should be referred to an expert multidisciplinary team for discussion

Recommendation 5 Children with any cancer from southern and south-eastern Brazilian families should be tested for the p.R337H Brazilian founder germline TP53 variant

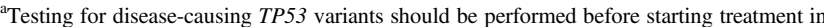
order to avoid in variant carriers, if possible, radiotherapy and genotoxic chemotherapy and to prioritise surgical treatments.

\section{Pre-symptomatic testing recommendations}

Recommendation 6 Adult first-degree relatives of individuals with germline diseasecausing TP53 variants should be offered testing for the same germline TP53 variant

Recommendation 7 The testing in childhood, from birth, of first-degree relatives of individuals with germline disease-causing TP53 variants should be systematically offered, if updated knowledge, based on databases and registries, shows that the variant can be considered as a high cancer risk TP53 variant conferring a high cancer risk in childhood:

- The index case has developed a childhood cancer; or

- Childhood cancers have been observed within the family; or

- This variant has already been detected in other families with childhood cancers; or

- This variant corresponds to a dominant-negative missense variant

Recommendation 8 The testing in childhood of first-degree relatives of individuals with germline disease-causing TP53 variants should not be systematically offered, if updated knowledge, based on databases and registries, shows that the variant can be considered as a low cancer risk TP53 variant and does not confer a high cancer risk in childhood:

- The index case has not developed a childhood cancer; and - Childhood cancers have not been observed within the family; and

- This variant has not already been reported in other families with childhood cancers; and

- This variant does not correspond to a dominant-negative missense variant

Recommendation 9 The testing in childhood of first-degree relatives of individuals with germline disease-causing TP53 variants should be discussed with their parents if cancers have occurred in early adulthood (before the age of 31 years) within the family, or if there is insufficient evidence in the databases or registries to determine the childhood cancer risk.

- This discussion should address the burden, and uncertain benefits, of surveillance in childhood, before a decision is made whether to test the child for germline disease-causing TP53 variants.

\section{Surveillance recommendations in carriers of germline disease-causing TP53 variants}

Recommendation 10 In children, clinical examination should be performed every 6 months, with specific attention to signs of virilization or early puberty, and measurement of blood pressure

In adults, clinical examination should be performed annually with specific attention, in patients who received radiotherapy, to occurrence of basal cell carcinomas within the radiotherapy field

Recommendation 11 In adults, WBMRI without gadolinium enhancement should be conducted annually

Recommendation 12 In individuals with high cancer risk TP53 variants or previously treated by chemotherapy or radiotherapy, WBMRI without gadolinium enhancement, should be conducted annually, from birth

Recommendation 13 In female individuals, breast MRI should be conducted annually, from 20 years until 65 years

Recommendation 14 In children, from birth, and in adolescents ( $<18$ years), abdominal ultrasound for the detection of adrenocortical carcinoma should be conducted at least every 6 months

Recommendation 15 In children, from birth, and in adolescents ( $<18$ years), when abdominal ultrasound does not allow a proper imaging of the adrenal
Table (continued)

\begin{tabular}{|c|c|}
\hline & $\begin{array}{l}\text { glands, measurement of urine steroids, for detection of ACC, should } \\
\text { probably be conducted at least every } 6 \text { months } \\
\text { In adults until } 50 \text { years, brain MRI should be conducted annually }\end{array}$ \\
\hline Recommendation 17 & $\begin{array}{l}\text { h cancer risk TP53 variants, brain MRI should } \\
\text { h, annually }\end{array}$ \\
\hline Recommendation 18 & $\begin{array}{l}\text { If surveillance includes brain MRI, at least the first (prevalence) scan } \\
\text { should be conducted using dedicated brain MRI with Gadolinium } \\
\text { enhancement }\end{array}$ \\
\hline Recommendation 19 & $\begin{array}{l}\text { In children, if surveillance includes annual brain MRI, this should } \\
\text { alternate with the WBMRI, so that the brain is imaged at least every } \\
6 \text { months }\end{array}$ \\
\hline Recommendation 20 & $\begin{array}{l}\text { Colonoscopy should be performed, from } 18 \text { years, every } 5 \text { years, only } \\
\text { if the carrier received abdominal radiotherapy for the treatment of a } \\
\text { previous cancer, or if there is a familial history of colorectal tumours } \\
\text { suggestive of an increased genetic risk }\end{array}$ \\
\hline
\end{tabular}

\section{Guideline summary: surveillance protocol in carriers of GERMLINE disease-causing TP53 variants}

\begin{tabular}{lllll}
\hline Exam & Periodicity & $\begin{array}{l}\text { Age } \\
\text { to start }\end{array}$ & Age to end Condition & Evidence $^{\mathrm{a}}$ \\
$\begin{array}{l}\text { Clinical examination } \\
\text { with, in children, }\end{array}$ & $\begin{array}{l}\text { Every } \\
\text { specific attention to }\end{array}$ & Birth & 17 years & Moderate \\
signs of virilisation \\
$\begin{array}{l}\text { or early puberty and } \\
\text { measurement of } \\
\text { blood pressure and, }\end{array}$ & & 18 years - & Moderate \\
$\begin{array}{l}\text { in patients who } \\
\text { received } \\
\text { radiotherapy, to } \\
\text { occurrence of basal } \\
\text { cell carcinomas } \\
\text { within the }\end{array}$ & & \\
radiotherapy field & & & \\
\end{tabular}

Whole-Body MRI

without gadolinium

Annual Birth -

TP53 variant ${ }^{\mathrm{b}}$ or patient previously

treated by

chemotherapy or

radiotherapy

Moderate

Breast MR

18 years -

Brain $\mathrm{MRI}^{\mathrm{c}}$

Annual

20 years Until

Annual Birth 18 years

High cancer risk

18 years Until

50 years

Abdominal

ultrasound

Urine steroids

Every

6 months

Every

Birth

Until

6 months

Birth

18 years

Until

When abdominal

ultrasound does not

allow a proper

imaging of the

adrenal glands

Colonoscopy

Every

18 years -

Only if the carrier received abdominal radiotherapy for
treatment of a

treatment of a
previous cancer or if there is a familiaT history of colorectal tumours suggestive

of an increased

genetic risk

${ }^{\mathrm{a}}$ This grading is based on published articles and expert consensus.

'A germline disease-causing TP53 variant should be considered as 'high risk' if the index case has developed a childhood cancer; or childhood cancers have been observed within the family; or this variant has already been detected in other families with childhood cancers; or this variant corresponds to a dominant-negative missense variant.

${ }^{\mathrm{c}}$ The first scan should be conducted with I.V. Gadolinium enhancement; in children, brain MRI should alternate with the Whole-Body MRI, so that the brain is imaged at least every 6 months. 
Acknowledgements The authors deeply thank Matt Bolz-Johnson and Tom Kenny for logistic support, coordination of the meetings and permanent commitment for the elaboration of these guidelines. They are grateful to the following colleagues for their critical review of the guidelines: Prof. Lennart Blomqvist, Diagnostic Imaging Unit, Karolinska University Hospital, Stockholm, Sweden; Dr. Laurence Brugières, Department of Paediatrics and Adolescents Oncology, Gustave Roussy, Villejuif, France; Dr. Suzette Delaloge, Department of Medical Oncology, Gustave Roussy, Villejuif, France; Dr. Christian Kratz, Paediatric Haematology and Oncology, Medical School, Hannover, Germany. They also acknowledge the following colleagues for fruitful discussions and suggestions: Prof. Stefan Aretz, University Hospital, Bonn, Germany; Dr. Ignacio Blanco, Institut Català de la Salut, Barcelona, Spain; Prof.Maurizio Genuardi, Fondazione Policlinico Universitario A. Gemelli, Roma, Italy; Prof. Elke Holinski Feder, Medizinisch Genetisches Zentrum, München, Germany; Prof. Jan Lubinski, Pomeranian Medical University - University Clinical Hospital, Szczecin, Poland; Dr. Hector Salvador, Hospital Sant Joan de Déu, Barcelona, Spain; Dr. Katharina Wimmer, Division of Human Genetics, Medical University Innsbruck, Innsbruck, Austria; Dr Emma Woodward, St Mary's Hospital, Central Manchester University Hospitals NHS Foundation Trust, Manchester, UK. This guideline has been supported by the European Reference Network on Genetic Tumour Risk Syndromes (ERN GENTURIS) - Project ID No 739547. ERN GENTURIS is partly co-funded by the European Union within the framework of the Third Health Programme 'ERN-2016 - Framework Partnership Agreement 2017-2021'.

The European Reference Network GENTURIS Nicoline Hoogerbrugge ${ }^{6}$, Marjolijn Ligtenberg ${ }^{6}$, Marleen Kets ${ }^{6,7}$, Rianne Oostenbrink ${ }^{8}$, Rolf Sijmons ${ }^{9}$, Gareth Evans ${ }^{10}$, Emma Woodward ${ }^{10}$, Marc Tischkowitz ${ }^{11}$ Eamonn Maher ${ }^{11}$, Rosalie E. Ferner ${ }^{12}$, Stefan Aretz ${ }^{13}$, Isabel Spier ${ }^{14}$, Verena Steinke-Lange ${ }^{14}$, Elke Holinski-Feder ${ }^{14}$, Evelin Schröck ${ }^{15}$, Thierry Frebourg ${ }^{16}$, Claude Houdayer ${ }^{16}$, Chrystelle Colas ${ }^{17}$, Pierre Wolkenstein ${ }^{18}$, Vincent Bours ${ }^{19}$, Eric Legius ${ }^{20}$, Bruce Poppe ${ }^{21}$, Kathleen Claes ${ }^{21}$, Robin de Putter ${ }^{21}$, Ignacio Blanco Guillermo ${ }^{22}$, Gabriel Capella ${ }^{22}$, Joan Brunet Vidal ${ }^{22}$, Conxi Lázaro ${ }^{22}$, Judith Balmaña $^{22}$, Hector Salvador Hernandez ${ }^{23}$, Carla Oliveira ${ }^{24}$, Manuel Teixeira $^{24}$, Svetlana Bajalica Lagercrantz ${ }^{25}$, Emma Tham ${ }^{25}$, Jan Lubinski ${ }^{26}$, Karolina Ertmanska ${ }^{26}$, Bela Melegh ${ }^{27}$, Mateja Krajc ${ }^{28}$, Ana Blatnik ${ }^{28}$, Sirkku Peltonen ${ }^{29}$, Marja Hietala ${ }^{29}$.

${ }^{6}$ Radboud University Medical Center, Nijmegen, The Netherlands; ${ }^{7} \mathrm{NKI}$ Antoni van Leeuwenhoek hospital, Amsterdam, The Netherlands; ${ }^{8}$ Erasmus Medical Center, Rotterdam, The Netherlands; ${ }^{9}$ University Medical Center, Groningen, The Netherlands; ${ }^{10}$ Genomic Medicine, Central Manchester Foundation Trust, Manchester, UK; ${ }^{11}$ Cambridge University Hospitals NHS Foundation Trust, Cambridge, UK; ${ }^{12}$ Guy's and St. Thomas' NHS Foundation Trust, London, UK; ${ }^{13}$ University Hospital Bonn, Bonn, Germany; ${ }^{14}$ Medizinisch Genetisches Zentrum, Munich, Germany; ${ }^{15}$ Hereditary Cancer Syndrome Center Dresden, Dresden, Germany; ${ }^{16}$ Rouen University Hospital, Rouen, France; ${ }^{17}$ Institut Curie, Paris, France; ${ }^{18}$ University Hospital Henri Mondor-National Referral Center, Créteil, France; ${ }^{19}$ University Hospital, Liege, Belgium; ${ }^{20}$ University Hospital Leuven, Leuven, Belgium; ${ }^{21}$ Ghent University Hospital, Ghent, Belgium; ${ }^{22}$ Hospital Universitari Germans Trias i Pujol y ICO Badalona, Institut Catala d'Oncologia, Barcelona, Spain; ${ }^{23}$ Hospital Sant Joan de Déu, Barcelona, Spain; ${ }^{24}$ Porto Comprehensive Cancer Center, Porto, Portugal; ${ }^{25}$ Karolinska University Hospital, Stockholm, Sweden; ${ }^{26}$ Pomeranian Medical University - University Clinical Hospital n1, Szczecin, Poland; ${ }^{27}$ University of Pécs, Pécs, Hungary; ${ }^{28}$ Institute of Oncology, Ljubljana, Slovenia; ${ }^{29}$ Turku University Hospital, Turku, Finland

\section{Compliance with ethical standards}

Conflict of interest The authors declare that they have no conflict of interest.

Disclaimer The content of these guidelines represents the views of the authors only and it is their sole responsibility; it cannot be considered to reflect the views of the European Commission and/or the Consumers, Health, Agriculture and Food Executive Agency (CHAFEA) or any other body of the European Union. The European Commission and the Agency do not accept any responsibility for use that may be made of the information it contains.

Publisher's note Springer Nature remains neutral with regard to jurisdictional claims in published maps and institutional affiliations.

Open Access This article is licensed under a Creative Commons Attribution 4.0 International License, which permits use, sharing, adaptation, distribution and reproduction in any medium or format, as long as you give appropriate credit to the original author(s) and the source, provide a link to the Creative Commons license, and indicate if changes were made. The images or other third party material in this article are included in the article's Creative Commons license, unless indicated otherwise in a credit line to the material. If material is not included in the article's Creative Commons license and your intended use is not permitted by statutory regulation or exceeds the permitted use, you will need to obtain permission directly from the copyright holder. To view a copy of this license, visit http://creativecommons. org/licenses/by/4.0/.

\section{References}

1. Li FP, Fraumeni JF Jr. Soft-tissue sarcomas, breast cancer, and other neoplasms. A familial syndrome? Ann Intern Med. 1969;71: 747-52.

2. Malkin D, Li FP, Strong LC, Fraumeni JF Jr, Nelson CE, Kim $\mathrm{DH}$, et al. Germ line p53 mutations in a familial syndrome of breast cancer, sarcomas, and other neoplasms. Science. 1990;250: $1233-8$.

3. Srivastava S, Zou ZQ, Pirollo K, Blattner W, Chang EH. Germline transmission of a mutated p53 gene in a cancer-prone family with Li-Fraumeni syndrome. Nature. 1990;348:747-9.

4. Gonzalez KD, Noltner KA, Buzin CH, Gu D, Wen-Fong CY, Nguyen VQ, et al. Beyond Li Fraumeni Syndrome: clinical characteristics of families with p53 germline mutations. J Clin Oncol. 2009;27:1250-6.

5. Ruijs MW, Verhoef S, Rookus MA, Pruntel R, van der Hout AH, Hogervorst FB, et al. TP53 germline mutation testing in 180 families suspected of Li-Fraumeni syndrome: mutation detection rate and relative frequency of cancers in different familial phenotypes. J Med Genet. 2010;47:421-8.

6. Bougeard G, Renaux-Petel M, Flaman JM, Charbonnier C, Fermey P, Belotti M, et al. Revisiting Li-Fraumeni syndrome from TP53 mutation carriers. J Clin Oncol. 2015;33:2345-52.

7. Varley JM, McGown G, Thorncroft M, James LA, Margison GP, Forster G. Are there low-penetrance TP53 Alleles? evidence from childhood adrenocortical tumors. Am J Hum Genet. 1999;65: 995-1006.

8. Wagner J, Portwine C, Rabin K, Leclerc JM, Narod SA, Malkin D. High frequency of germline p53 mutations in childhood adrenocortical cancer. J Natl Cancer Inst. 1994;86:1707-10. 16

9. Wasserman JD, Novokmet A, Eichler-Jonsson C, Ribeiro RC, Rodriguez-Galindo C, Zambetti GP, et al. Prevalence and 
functional consequence of TP53 mutations in pediatric adrenocortical carcinoma: a children's oncology group study. J Clin Oncol. 2015;33:602-9.

10. Tabori U, Shlien A, Baskin B, Levitt S, Ray P, Alon N, et al. TP53 alterations determine clinical subgroups and survival of patients with choroid plexus tumors. J Clin Oncol. 2010;28: 1995-2001.

11. Hettmer S, Archer NM, Somers GR, Novokmet A, Wagers AJ, Diller L, et al. Anaplastic rhabdomyosarcoma in TP53 germline mutation carriers. Cancer. 2014;120:1068-75.

12. Fortuno C, James PA, Spurdle AB. Current review of TP53 pathogenic germline variants in breast cancer patients outside $\mathrm{Li}$ Fraumeni syndrome. Hum Mutat. 2018;39:1764-73.

13. Gonzalez KD, Buzin CH, Noltner KA, Gu D, Li W, Malkin D, et al. High frequency of de novo mutations in Li-Fraumeni syndrome. J Med Genet. 2009;46:689-93.

14. Renaux-Petel M, Charbonnier F, Théry JC, Fermey P, Lienard G, Bou J, et al. Contribution of de novo and mosaic TP53 mutations to Li-Fraumeni syndrome. J Med Genet. 2018;55:173-80.

15. Holmfeldt L, Wei L, Diaz-Flores E, Walsh M, Zhang J, Ding L, et al. The genomic landscape of hypodiploid acute lymphoblastic leukemia. Nat Genet. 2013;45:242-52.

16. Qian M, Cao X, Devidas M, Yang W, Cheng C, Dai Y, et al. TP53 Germline variations influence the predisposition and prognosis of B-cell acute lymphoblastic leukemia in children. J Clin Oncol. 2018;36:591-9.

17. Waszak SM, Northcott PA, Buchhalter I, Robinson GW, Sutter C, Groebner S, et al. Spectrum and prevalence of genetic predisposition in medulloblastoma: a retrospective genetic study and prospective validation in a clinical trial cohort. Lancet Oncol. 2018;19:785-98.

18. Kato S, Han SY, Liu W, Otsuka K, Shibata H, Kanamaru R, et al. Understanding the function-structure and function-mutation relationships of p53 tumor suppressor protein by high-resolution missense mutation analysis. Proc Natl Acad Sci USA. 2003;100:8424-9.

19. Zerdoumi Y, Lanos R, Raad S, Flaman JM, Bougeard G, Frebourg $\mathrm{T}$, et al. Germline TP53 mutations result into a constitutive defect of p53 DNA binding and transcriptional response to DNA damage. Hum Mol Genet. 2017;26:2591-602.

20. Giacomelli AO, Yang X, Lintner RE, McFarland JM, Duby M, Kim J, et al. Mutational processes shape the landscape of TP53 mutations in human cancer. Nat Genet. 2018;50:1381-7.

21. Kotler E, Shani O, Goldfeld G, Lotan-Pompan M, Tarcic O, Gershoni A, et al. A systematic p53 mutation library links differential functional impact to cancer mutation pattern and evolutionary conservation. Mol Cell. 2018;71:178-90.

22. Coombs CC, Zehir A, Devlin SM, Kishtagari A, Syed A, Jonsson P. Therapy-related clonal hematopoiesis in patients with nonhematologic cancers is common and associated with adverse clinical outcomes. Cell Stem Cell. 2017;21:374-82.e4.

23. Weber-Lassalle K, Harter P, Hauke J, Ernst C, Kommoss S, Marmé $\mathrm{F}$, et al. Diagnosis of Li-Fraumeni syndrome: differentiating TP53 germline mutations from clonal hematopoiesis: results of the observational AGO-TR1 trial. Hum Mutat. 2018;39:2040-6.

24. Weitzel JN, Chao EC, Nehoray B, Van Tongeren LR, LaDuca H, Blazer KR, et al. Somatic TP53 variants frequently confound germ-line testing results. Genet Med. 2018;20:809-16.

25. Chen S, Liu Y. p53 involvement in clonal hematopoiesis of indeterminate potential. Curr Opin Hematol. 2019;4:235-40.

26. Chompret A, Brugières L, Ronsin M, Gardes M, DessarpsFreichey F, Abel A, et al. P53 germline mutations in childhood cancers and cancer risk for carrier individuals. $\mathrm{Br} \mathrm{J}$ Cancer. 2000;82:1 932-7.
27. Mai PL, Best AF, Peters JA, DeCastro RM, Khincha PP, Loud JT, et al. Risks of first and subsequent cancers among TP53 mutation carriers in the National Cancer Institute Li-Fraumeni syndrome cohort. Cancer. 2016;122:3673-81.

28. Amadou A, Waddington Achatz MI, Hainaut P. Revisiting tumor patterns and penetrance in germline TP53 mutation carriers: temporal phases of Li-Fraumeni syndrome. Curr Opin Oncol. 2018;30:23-29.

29. de Andrade KC, Frone MN, Wegman-Ostrosky T, Khincha PP, Kim J, Amadou A, et al. Variable population prevalence estimates of germline TP53 variants: a gnomAD-based analysis. Hum Mutat. 2019;40:97-105.

30. Shin SJ, Dodd-Eaton EB, Peng G, Bojadzieva J, Chen J, Amos CI, et al. Penetrance of different cancer types in families with $\mathrm{Li}$ Fraumeni syndrome: a validation study using multi-center cohorts. Cancer Res. 2019. https://doi.org/10.1158/0008-5472.CAN-190728.

31. Wong P, Verselis SJ, Garber JE, Schneider K, DiGianni L, Stockwell DH, et al. Prevalence of early onset colorectal cancer in 397 patients with classic Li-Fraumeni syndrome. Gastroenterology. 2006;130:73-9.

32. Yurgelun MB, Masciari S, Joshi VA, Mercado RC, Lindor NM, et al. Germline TP53 mutations in patients with early-onset colorectal cancer in the colon cancer family registry. JAMA Oncol. 2015;1:214-21.

33. MacFarland SP, Zelley K, Long JM, McKenna D, Mamula P, Domchek SM, et al. earlier colorectal cancer screening may be necessary in patients with Li-Fraumeni syndrome. Gastroenterology. 2019;15:273-4.

34. Figueiredo BC, Sandrini R, Zambetti GP, Pereira RM, Cheng C, Liu W, et al. Penetrance of adrenocortical tumours associated with the germline TP53 R337H mutation. J Med Genet. 2006;43:91-6.

35. Achatz MI, Olivier M, Le Calvez F, Martel-Planche G, Lopes A, Rossi BM, et al. The TP53 mutation, $\mathrm{R} 337 \mathrm{H}$, is associated with Li-Fraumeni and Li-Fraumeni-like syndromes in Brazilian families. Cancer Lett. 2007;245:96-102.

36. Palmero EI, Schüler-Faccini L, Caleffi M, Achatz MI, Olivier M, Martel-Planche G, et al. Detection of R337H, a germline TP53 mutation predisposing to multiple cancers, in asymptomatic women participating in a breast cancer screening program in Southern Brazil. Cancer Lett. 2008;261:21-5.

37. Kasper E, Angot E, Colasse E, Nicol L, Sabourin JC, Adriouch S, et al. Contribution of genotoxic anticancer treatments to the development of multiple primary tumours in the context of germline TP53 mutations. Eur J Cancer. 2018;101:254-62.

38. Villani A, Shore A, Wasserman JD, Stephens D, Kim RH, Druker H, et al. Biochemical and imaging surveillance in germline TP53 mutation carriers with Li-Fraumeni syndrome: 11 year follow-up of a prospective observational study. Lancet Oncol. 2016;17:1295-305.

39. Kratz CP, Achatz MI, Brugières L, Frebourg T, Garber JE, Greer $\mathrm{MC}$, et al. Cancer screening recommendations for individuals with Li-Fraumeni syndrome. Clin Cancer Res. 2018;23:e38-e45.

40. Ballinger ML, Best A, Mai PL, Khincha PP, Loud JT, Peters JA, et al. Baseline surveillance in Li-Fraumeni syndrome using wholebody magnetic resonance imaging: a meta-analysis. JAMA Oncol. 2017;3:1634-9.

41. Caron O, Frebourg T, Benusiglio PR, Foulon S, Brugières L. Lung adenocarcinoma as part of the Li-Fraumeni syndrome spectrum: preliminary data of the LIFSCREEN randomized clinical trial. JAMA Oncol. 2017;3:1736-7.

42. Ruijs MWG, Loo CE, van Buchem CAJM, Bleiker EMA, Sonke GS. Surveillance of Dutch patients with Li-Fraumeni syndrome: the LiFe-Guard study. JAMA Oncol. 2017;3:1733-4.

43. Saya S, Killick E, Thomas S, Taylor N, Bancroft EK, Rothwell J, et al. Baseline results from the UK SIGNIFY study: a whole-body 
MRI screening study in TP53 mutation carriers and matched controls. Fam Cancer. 2017;16:433-40.

44. Bojadzieva J, Amini B, Day SF, Jackson TL, Thomas PS, Willis BJ, et al. Whole body magnetic resonance imaging (WBMRI) and brain MRI baseline surveillance in TP53 germline mutation carriers: experience from the Li-Fraumeni Syndrome Education and Early Detection (LEAD) clinic. Fam Cancer 2018;17:287-94.

45. O'Neill AF, Voss SD, Jagannathan JP, Kamihara J, Nibecker C, Itriago-Araujo $\mathrm{E}$, et al. Screening with whole-body magnetic resonance imaging in pediatric subjects with Li-Fraumeni syndrome: A single institution pilot study. Pediatr. Blood Cancer. 2018;65. https://doi.org/10.1002/pbc.26822.

46. Paixao D, Guimaraes MD, de Andrade KC, Nobrega AF, Chojniak R, Achatz MI. Whole-body magnetic resonance imaging of Li-Fraumeni syndrome patients: observations from a two rounds screening of Brazilian patients. Cancer Imaging. 2018;18:27.

47. Layne KA, Dargan PI, JRH Archer, Wood DM. Gadolinium deposition and the potential for toxicological sequelae-a literature review of issues surrounding gadolinium-based contrast agents. Br J Clin Pharm. 2018;84:2522-34. 